\title{
WEYL-MECHANICAL SYSTEMS ON GENERALIZED (PARA)-KÄHLER SPACE FORM
}

\section{ZEKI KASAP}

Department of Elementary Mathematics

Faculty of Education

Pamukkale University

Denizli

Turkey

e-mail: zekikasap@hotmail.com

\begin{abstract}
Euler-Lagrange and Hamilton equations on Kähler-Weyl manifolds were presented and the para-complex mathematical aspects of Lagrangian and Hamilton operator, dynamic equation, the action functional, Lagrangian and Hamilton's principle and equations and so on were given. The most important result revealed by this study, how to find the Lagrangian and Hamiltonian equations of motion without using the dynamic equations. For this, theorems were used as an alternative method of finding equations. As a result of this study, Weyl-Euler-Lagrange and Weyl-Hamilton partial differential equations were obtained for movement of objects on Kähler-Weyl manifolds.
\end{abstract}

2010 Mathematics Subject Classification: 30C20, 32Q15, 32Q20, 34B20, 51P05, 53C15, 58J60.

Keywords and phrases: dynamics equation, conformal geometry, generalized (para)Kählerian space form, Weyl geometry, Lagrangian, Hamiltonian.

Received June 17, 2020; Revised August 6, 2020

(C) 2020 Scientific Advances Publishers 


\section{Introduction}

A Kähler manifold is a manifold with three mutually compatible structures, which are complex, Riemann and symplectic structure. It is well known that a conformal structure $C$ on the manifold $M$ is the collection of all conformally equivalent Riemannian metrics given on $M$. A Weyl structure $\nabla$ on a conformal manifold $(M, C)$ is a torsion-free linear connection $\nabla^{J}$ preserving the conformal structure $C$. Generalizing the usual Euler-Lagrange and Hamilton equations can be found with complex and to the para-complex settings to the context of a generalized (para)-Kählerian space. Also, using the mechanical principle, the theory of modern differential geometry and advanced calculus, Lagrangian and Hamiltonian mechanics for mechanical systems can be extended to Kähler-Weyl manifolds.

Gilkey and Nikcević [1] submitted in both complex and para-complex categories and examine (para)-Kähler Weyl structures in both geometric and in the algebraic settings. León and Rodrigues [2] offered a geometrical approach of Lagrangian and Hamiltonian formalisms involving higher order derivatives. Vries [3] shown that the Hamiltonian and Lagrangian motion equations have a very simple interpretation in relativistic quantum mechanics. Tekkoyun [4] presented that paracomplex analogue of the Euler-Lagrange and Hamilton equations was obtained in the framework of para-Kählerian manifold. Tekkoyun and Sari [5] introduced bi-para-complex analogue of Lagrangian and Hamiltonian systems. Tekkoyun and Yayli [6] presented generalizedquaternionic Kählerian analogue of Lagrangian and Hamiltonian mechanical systems. Gilkey and Nikčević [7] gave an elementary proof of the fact that any 4-dimensional para-Hermitian manifold admits a unique para-Kähler-Weyl structure. Ganchev and Mihova [8] submitted warped product Kähler manifolds that they used as an underlying manifold an alpha-Sasakian manifold. Bejan and Chiriac [9] introduced a new 
covariant differential ${ }^{A} \nabla$ adapted to an almost paracontact structure. Cherney et al. [10] presented a gauge invariant generalization of Maxwell's equations and $p$-form electromagnetism to a Kähler spacetime. Sun and Wang [11] defined a class of geometric flows on a complete Kähler manifold to unify some physical and mechanical models. Freed [12] gave an intrinsic definition of the special geometry which arises in global $N=2$ supersymmetry in four dimensions. Tyurin [13] showed a new approach to geometrical quantization procedure. Lyakhovich and Sharapov [14] examined that necessary and sufficient conditions were established for the second class constraint surface to be (an almost) Kähler manifold. Rongye [15] discussed Newtonian mechanics on a Kähler manifold and also gave the complex mathematical aspects of Newton's law. Kasap and Tekkoyun [16] introduced Lagrangian and Hamiltonian formalism for mechanical systems using para/pseudo-Kähler manifolds. Petrović and Velimirović [17] defined a new class of generalized para-Kähler spaces by using two different kinds of covariant derivatives. Petrović [18] defined generalized hyperbolic Kähler spaces as a particular case of Eisenhart's generalized Riemannian spaces.

\section{Preliminaries}

Definition 1 ([19]). A conformal map is a function which preserves angles. A conformal manifold is a differentiable manifold equipped with an equivalence class of (pseudo) Riemann metric tensors, in which two metrics $g^{\prime}$ and $g$ are equivalent if and only if

$$
g^{\prime}=\Psi^{2} g
$$

where $\Psi>0$ is a smooth positive function. An equivalence class of such metrics is known as a conformal class. A manifold with a conformal structure is called a conformal manifold. 
Definition 2 ([19]). Two Riemann metrics $g_{1}$ and $g_{2}$ on $M$ are said to be conformally equivalent if there exists a smooth function $f: M \rightarrow \mathbb{R}$ with

$$
e^{f} g_{1}=g_{2} \text {. }
$$

If the metrics $g_{1}$ and $g_{2}$ are conformally equivalent, we will write $g_{1} \sim g_{2}$.

Definition 3 ([19]). Let $M$ be an $n$-dimensional smooth manifold. A pair $(M, C)$, is said to be a conformal structure on $M$ is an equivalence class $C$ of Riemann metrics on $M$, is called a conformal structure.

Theorem 1 (See [20]). Let $\nabla$ be a connection on $M$ and $g \in C$ be a fixed metric. $\nabla$ is compatible with $(M, C)$ if and only if there exists a 1-form $\omega$ with $\nabla_{X} g+\omega(X) g=0$.

Definition 4 ([21]). A compatible torsion-free connection is called a Weyl connection. The triple $(M, C, \nabla)$ is a Weyl structure.

Theorem 2 ([22]). To each metric $g \in C$ and 1-form $\omega$, there corresponds a unique Weyl connection $\nabla$ satisfying $\nabla_{X} g+\omega(X) g=0$.

Definition 5 ([22]). If $F:\{1$-forms on $M\} \times C \rightarrow\{$ Weyl connections $\}$ is a function defined $F(g, \omega)=\nabla$, where $\nabla$ is the connection guaranteed by Theorem 2 we say that $\nabla$ corresponds to $(g, \omega)$.

A function $F$ defined in Definition 5 by has the properties:

Proposition 1 ([22]). (1) $F$ is surjective.

(2) $F(g, \omega)=F\left(e^{f} g, \eta\right)$ iff $\eta=\omega-d f$. So

$$
F\left(e^{f} g\right)=F(g)-d f
$$


Note that a Riemann metric $g$ and a one-form $\omega$ determine a Weyl structure, namely $F: C \rightarrow \wedge^{1} M$, where $C$ is a conformal structure and equivalence class of $g$ and $F\left(e^{f} g\right)=\omega-d f$.

Proof. We will show that $\nabla_{X} g$ is equal to the $\nabla_{X}\left(e^{f} g\right)$. Suppose $F(g, \omega)=F\left(e^{f} g, \eta\right)=\nabla$. We have

$$
\begin{aligned}
\nabla_{X}\left(e^{f} g\right)+\eta(X) e^{f} g & =X\left(e^{f}\right) g+e^{f} \nabla_{X} g+\eta(X) e^{f} g \\
& =\nabla f(X) e^{f} g+e^{f} \nabla_{X} g+\eta(X) e^{f} g=0 .
\end{aligned}
$$

Therefore $\nabla_{X} g=-(d f(X)+\eta(X))$. On the other hand $\nabla_{X} g+\omega(X) g=0$. Therefore $\omega=\eta+d f$. Set $\nabla=F(g, \omega)$ and show that $\nabla=F\left(e^{f} g, \eta\right)$ and $\nabla_{X}\left(e^{f} g\right)+\eta(X) e^{f} g=0$. To calculate

$$
\begin{aligned}
\nabla_{X}\left(e^{f} g\right)+\eta(X) e^{f} g & =e^{f} d f(X) g+e^{f} \nabla_{X} g+(\omega(X)-d f(X)) e^{f} g \\
& =e^{f}\left(\nabla_{X} g+\omega(X) g\right)=0 .
\end{aligned}
$$

Such a geometric structure was introduced by Weyl in 1922 in an attempt to unify gravity with electromagnetism [23]. Einstein believed till the end of his life in 1955 that the non-symmetric gravitational theory will be the right choice for the so-called theory of everything. But Eisenhart's generalized Riemannian spaces are important, because these spaces were fundamental in unifying the theory of gravitation and electromagnetism by Einstein who considered a manifold with a non-symmetric basic tensor [24]. 


\section{Pseudo-Riemannian Weyl Geometry}

Definition 6 ([25]). A pair $(J, g)$ consisting of a complex structure $J$ on $M$ and a Hermitian metric $g$ in the tangent bundle $T M$, a Riemannian metric $g$ that is invariant under $J, g(J X, J Y)=g(X, Y)$ for any vector fields $X$ and $Y$ on $M$. A Hermitian structure specifies in any tangent space $T_{p} M$ the structure of a Hermitian vector space. A manifold with a Hermitian structure is called a Hermitian manifold.

Definition 7 ([25]). The most important class of Hermitian manifolds are Kähler manifolds. These are Hermitian manifolds for which the Hermitian form $\omega$ is closed: $d \omega=0$. In this case the form $\omega$ is called a Kähler form. An almost Hermitian manifold whose associated can a form be of type $(1,1)$ is closed is naturally called an almost Kähler manifold. Any symplectic manifold admits a compatible almost complex structure making it into an almost Kähler manifold.

Let $\nabla$ be a torsion-free connection on a pseudo-Riemannian manifold $(M, g)$ of even dimension $n=2 m \geq 4$. The triple $(M, g, \nabla)$ is said to be a Weyl structure if there exists a smooth 1-form $\varnothing$ so that $\nabla g=-2 \varnothing \otimes g$.

Let $n=2 m \geq 4$. A triple $\left(M, g, J_{ \pm}\right)$is said to be an almost pseudo/para-Hermitian manifold with an almost pseudo/para-complex structure $J_{ \pm}$. Here, $J_{+}$is called para and $J_{-}$is called pseudo, if $g$ is a pseudo/para-Riemannian metric on $M$ of neutral signature $(m, m)$ and if $J_{ \pm}$is an endomorphism of the tangent bundle $T M$ so that $J_{ \pm}^{2}=I d$ and so that $J_{ \pm}^{*} g=\mp g ;\left(M, g, J_{ \pm}\right)$is said to be pseudo/para-Hermitian with an integrable complex structure $J_{ \pm}$if the pseudo/para-Nijenhuis tensor

$$
N_{ \pm}(x, y)=[x, y] \mp J_{ \pm}\left[J_{ \pm} x, y\right] \mp J_{ \pm}\left[x, J_{ \pm} y\right] \pm\left[J_{ \pm} x, J_{ \pm} y\right] .
$$


Let $\left(M, g, J_{ \pm}, \nabla\right)$ be an almost pseudo/para-Hermitian Weyl manifold. If $\nabla\left(J_{ \pm}\right)=0$, then one says that this is a (para)-Kähler Weyl manifold. Note that necessarily $J_{ \pm}$is integrable in this setting. $J_{ \pm}$is integrable means there exist local coordinates $\left(x^{1}, \ldots, x^{\bar{m}}, y^{1}, \ldots, y^{\bar{m}}\right)$ so $J_{ \pm}\left(\partial x_{i}\right)=\partial y_{i}$ and $J_{ \pm}\left(\partial y_{i}\right)= \pm \partial x_{i}$, i.e., the Nijenhuis tensor $N_{ \pm}=0$. Let $\Omega_{ \pm}(x, y):=g\left(x, J_{ \pm} y\right)$ be the (para)-Kähler form. The following conditions are equivalent and if any is satisfied $\left(M, g, \nabla, J_{ \pm}\right)$is said to be Kähler. $\nabla^{g} J_{ \pm}=0, d \Omega_{ \pm}=0$ and $J_{ \pm}$is integrable and given $p \in M$.

Theorem 3 ([26]). (a) If

$$
\widetilde{g}=e^{2 f} g
$$

is a conformally equivalent positive definite metric, then $(M, \widetilde{g}, \nabla)$ is a Weyl manifold with associated 1-form $\widetilde{\varnothing}=\varnothing-d f$. If $\nabla g$ is the LeviCivita connection, we may then express $\nabla=\nabla^{\varnothing}$ in the form:

$$
\nabla_{x}^{\varnothing} y=\nabla_{x}^{g} y+\varnothing(x) y+\varnothing(y) x-g(x, y) \varnothing^{*}
$$

where $\widetilde{\varnothing}^{*}$ is the dual vector field. Thus $\varnothing$ determines $\nabla$. Conversely, if $\varnothing$ is given and if we use Equation (8) to define $\nabla$, then $\nabla$ is a Weyl connection with associated 1-form $\varnothing$.

(b) $\left(M, g, J_{ \pm}, \nabla\right)$ is Kähler-Weyl and $m \geq 6$, then the Weyl structure is trivial, i.e., there is a conformally equivalent metric so that $\left(M, \widetilde{g}, J_{ \pm}\right)$ is Kähler.

(c) Let $m=4$. If $\left(M, g, J_{ \pm}\right)$is pseudo/para-Hermitian, then there is a unique torsion free connection $\nabla$ so $\left(M, g, J_{ \pm}, \nabla\right)$ is Kähler Weyl; here $\varnothing= \pm \frac{1}{2} J_{ \pm}^{*} \delta \Omega_{ \pm}$. 
A symplectic form on a smooth manifold $M$ is a smooth closed 2-form omega on $M$ which is nondegenerate such that at every point $p$, the alternating bilinear form $\omega_{p}$ on the tangent space $T_{p} M$ is nondegenerate.

\section{Generalized (Para)-Kähler-Weyl Space Form}

Definition 8 ([21, 28]). A Kähler form $\omega$ is a real closed nondegenerate symplectic two-form for which $\omega(X, J X)>0$ for nonzero tangent vectors $X \in T_{p} M$. It must also satisfy $\omega(J X, J Y)=\omega(X, Y)$, where $J$ is the almost complex structure induced by multiplication by almost complex structure $\left(\mathbf{i}^{2}=-1\right)$. That is,

$$
J_{ \pm}\left(\frac{\partial}{\partial x_{i}}\right)=\frac{\partial}{\partial y_{i}}, \quad J_{ \pm}\left(\frac{\partial}{\partial y_{i}}\right)= \pm \frac{\partial}{\partial x_{i}}, i=1,2
$$

The dual endomorphism $J_{ \pm}^{*}$ of $T_{P}^{*}(M)$ at any point $P$ of a manifold $M$ satisfies $J_{ \pm}^{* 2}= \pm I d$ and it is defined by

$$
J_{ \pm}^{*}\left(d x_{i}\right)=d y_{i}, \quad J_{ \pm}^{*}\left(d y_{i}\right)= \pm d x_{i}, i=1,2 .
$$

Proposition 2 ([29]). (a) A manifold with a Weyl structure is known as a Weyl manifold. Let's we use (7) to get base structures. If we rewrite Equation (9) with conformal structure, we obtain the following equations:

$$
J_{ \pm}\left(\frac{\partial}{\partial x_{i}}\right)=e^{2 f} \frac{\partial}{\partial y_{i}}, \quad J_{ \pm}\left(\frac{\partial}{\partial y_{i}}\right)= \pm e^{-2 f} \frac{\partial}{\partial x_{i}},
$$

that are base structures for Euler-Lagrange equations, where $J_{ \pm}$is a conformal (para)-complex structure to an integrable almost (para)complex $J_{ \pm}$given as the above. $f$ second structure was chosen the minus sign. Because the condition of the structure required to provide $J_{ \pm}^{2}= \pm I d$. 
(b) Similarly $J_{ \pm}^{*}$ the structures being the dual of $J_{ \pm}$structures are given with conformal structure as follows:

$$
J_{ \pm}^{*}\left(d x_{i}\right)=e^{2 f} d y_{i}, \quad J_{ \pm}^{*}\left(d y_{i}\right)= \pm e^{-2 f} d x_{i},
$$

are base structures for Hamilton equations. We continue our studies thinking of the $\left(M, g, \nabla, J_{ \pm}\right)$and $\left(M, g, \nabla, J_{ \pm}^{*}\right)$ instead of the almost para/pseudo-Kähler Weyl manifolds $\left(M, g, \nabla, J_{ \pm}\right)$, and also its dual $\left(M, g, \nabla, J_{ \pm}^{*}\right)$, respectively.

\section{Proof.}

(a) Let's examine these structures holomorphic property

$$
\begin{aligned}
J_{ \pm}^{2}\left(\frac{\partial}{\partial x_{i}}\right) & =J_{ \pm} \circ J_{ \pm}\left(\frac{\partial}{\partial x_{i}}\right)=J_{ \pm}\left(e^{2 f} \frac{\partial}{\partial y_{i}}\right) \\
& =e^{2 f} J_{ \pm}\left(\frac{\partial}{\partial y_{i}}\right)= \pm e^{2 f} e^{-2 f} \frac{\partial}{\partial x_{i}}= \pm \frac{\partial}{\partial x_{i}}, \\
J_{ \pm}^{2}\left(\frac{\partial}{\partial y_{i}}\right) & =J_{ \pm} \circ J_{ \pm}\left(\frac{\partial}{\partial y_{i}}\right)=J_{ \pm}\left( \pm e^{-2 f} \frac{\partial}{\partial x_{i}}\right) \\
& = \pm e^{-2 f} J_{ \pm}\left(\frac{\partial}{\partial x_{i}}\right)= \pm e^{-2 f} e^{2 f} \frac{\partial}{\partial y_{i}}= \pm \frac{\partial}{\partial y_{i}} .
\end{aligned}
$$

(b) Similarly,

$$
\begin{aligned}
J_{ \pm}^{* 2}\left(d x_{i}\right) & =J_{ \pm}^{*} \circ J_{ \pm}^{*}\left(d x_{i}\right)=J_{ \pm}^{*}\left(e^{2 f} d y_{i}\right) \\
& =e^{2 f} J_{ \pm}^{*}\left(d y_{i}\right)= \pm e^{2 f} e^{-2 f} d x_{i}= \pm d x_{i}, \\
J_{ \pm}^{* 2}\left(d y_{i}\right) & =J_{ \pm}^{*} \circ J_{ \pm}^{*}\left(d y_{i}\right)=J_{ \pm}^{*}\left( \pm e^{-2 f} d x_{i}\right) \\
& = \pm e^{-2 f} J_{ \pm}^{*}\left(d x_{i}\right)= \pm e^{2 f} e^{-2 f} d y_{i}= \pm d y_{i} .
\end{aligned}
$$

As we have seen, $J_{ \pm}^{2}= \pm I$ are the para(pseudo) complex structures type. 


\section{1. (Euler)-Lagrange and Hamilton dynamics equations}

Lagrange and Hamilton dynamic equations ([27]) were introduced by Klein in 1962 and they are shown as follows.

(a) Lagrange dynamic equation: Let $M$ be an $n$-dimensional manifold and $T M$ be its tangent bundle with canonical projection $\tau_{M}: T M \rightarrow M$. TM is called the phase space of velocities of the base manifold $M$. Let $L: T M \rightarrow \mathbb{R}$ be a differentiable function on $T M$ called a Lagrangian function. We consider the closed 2 -form on $T M$ given by $\Phi_{L}=-d\left(d_{J} L\right)$. Lagrange dynamic equation is

$$
i_{\xi} \Phi_{L}=d E_{L}
$$

Then $\xi$ is a vector field, we shall see that (15) under a certain condition on $\xi$ is the intrinsical expression of the Euler-Lagrange equations of motion. This equation is named as Lagrange dynamical equation. We shall see that for motion in a potential, $E_{L}=V(L)-L$ is an energy function and $V=J(\xi)$ a Liouville vector field. Here $d E_{L}$ denotes the differential of $E$. The triple $\left(T M, \Phi_{L}, \xi\right)$ is known as Lagrangian system on the tangent bundle $T M$. If it is continued the operations on (15) for any coordinate system $\left(q^{i}(t), p_{i}(t)\right)$, infinite dimension Lagrange's equation is obtained the form below:

$$
\frac{\partial}{\partial t}\left(\frac{\partial L}{\partial \dot{q}^{i}}\right)=\frac{\partial L}{\partial q^{i}}, \frac{\partial q^{i}}{\partial t}=\dot{q}^{i}, i=1, \ldots, n \quad \text { (a proof see in [30]) }
$$

(b) Hamilton dynamic equation: Let $M$ is a base manifold and its cotangent manifold $T^{*} M$. By a symplectic form we mean a 2 -form $\Phi$ on $T^{*} M$. Let $\left(T^{*} M, \Phi\right)$ be a symplectic manifold. A vector field $X_{H}: T^{*} M \rightarrow T T^{*} M$ is called Hamiltonian function if there exists a $C^{1}$ 
function $H: T^{*} M \rightarrow \mathbb{R}$ such that Hamilton dynamical equation is determined by

$$
i_{X_{H}} \Phi=d H
$$

We say that $X_{H}$ is a locally Hamiltonian vector field. The 2 -form $\Phi$ is closed and it is a canonical symplectic form given by $\Phi=-d \Omega$, where $\Omega=J^{*}(\omega), J^{*}$ is a dual of $J$, and $\omega$ is a 1 -form on $T^{*} M$. The triple $\left(T^{*} M, \Phi, X_{H}\right)$ is called a Hamiltonian system which is defined on the cotangent bundle $T^{*} M$. In Darboux coordinates $\left(q^{i}(t), p_{i}(t)\right)$ we have $\omega=d q^{i} \wedge d p_{i}$ and $X_{H}=\frac{\partial H}{\partial p_{i}} \frac{\partial}{\partial q^{i}}-\frac{\partial H}{\partial q^{i}} \frac{\partial}{\partial p_{i}}$ so that the integral curves $\left(q^{i}(t), p_{i}(t)\right)$ is an integral curve of $X_{H}$ satisfy the Hamilton equations

$$
\frac{d q^{i}}{d t}=\frac{\partial H}{\partial p_{i}}, \frac{d p_{i}}{d t}=-\frac{\partial H}{\partial q^{i}} \quad \quad \text { (proof see [30]) }
$$

\section{Weyl-Euler-Lagrangian Mechanics Systems}

Theorem 4. Let $J$ be a para-complex or complex structure on the tangent space $T_{p}(M)$ of manifold $M$ at the point $p \in M$. If the $J$ base structure is known or can be found, the Euler-Lagrange equations of motion can be obtained without using the dynamic equation (15) for mechanical systems on the defined manifold of moving objects. The way to find the time-dependent Euler-Lagrange motion equations that can be obtained for mechanical systems without any calculations. In short, if the base structure is $J\left(\frac{\partial}{\partial x_{1}}\right)=\frac{\partial}{\partial x_{2}}$, the equation is $\frac{\partial}{\partial t}\left(\frac{\partial L}{\partial x_{2}}\right)=\frac{\partial L}{\partial x_{1}}$ or $\frac{\partial}{\partial t}$ $\left(\frac{\partial L}{\partial x_{2}}\right)-\frac{\partial L}{\partial x_{1}}=0$. The transactions made here are as follows: (a) The Lagrangian function " $L$ " is added to the base vectors of defined space 
$\frac{\partial}{\partial x_{1}} \rightarrow \frac{\partial L}{\partial x_{1}}$ and their location changes $J\left(\frac{\partial L}{\partial x_{2}}\right)=\frac{\partial L}{\partial x_{1}}$, (b) Instead of the "J" holomorphic structure, $\frac{\partial}{\partial t}$ expressing the derivative by time is introduced $\frac{\partial}{\partial t}\left(\frac{\partial L}{\partial x_{2}}\right)=\frac{\partial L}{\partial x_{1}}$.

For example: Base structures are (1) $J\left(\frac{\partial}{\partial z^{i}}\right)=-\mathbf{j} \frac{\partial}{\partial z^{i}}$ and (2) $J\left(\frac{\partial}{\partial \bar{z}^{i}}\right)=\mathbf{j} \frac{\partial}{\partial \bar{z}^{i}}$. Equations are (1) $\mathbf{j} \frac{\partial}{\partial t}\left(\frac{\partial L}{\partial z^{i}}\right)+\frac{\partial L}{\partial z^{i}}=0$ and (2) $\mathbf{j} \frac{\partial}{\partial t}$ $\left(\frac{\partial L}{\partial \dot{z}^{i}}\right)+\frac{\partial L}{\partial \dot{z}^{i}}=02[4]$.

Example. The motion equations for (11) are, according to Hamilton dynamical equation and Theorem 4 for a conformal (para)-complex structure to an integrable almost (para)-complex $J_{ \pm}$,

$$
\begin{gathered}
\frac{\partial}{\partial t}\left(e^{2 f} \frac{\partial L}{\partial y_{i}}\right)-\frac{\partial L}{\partial x_{i}}=0, \\
\frac{\partial}{\partial t}\left(e^{-2 f} \frac{\partial L}{\partial x_{i}}\right) \mp \frac{\partial L}{\partial y_{i}}=0 .
\end{gathered}
$$

The differential equations (19) are called conformal Weyl-EulerLagrange equations on a generalized (para)-Kählerian space form $\left(M, g, \nabla, J_{ \pm}\right)$. Finally, it may be said that the triple $\left(M, \Phi_{L}, \xi\right)$ is conformal Weyl-Euler-Lagrange mechanical system on a generalized (para)-Kählerian manifold of constant $J_{ \pm}$-sectional curvature $\left(M, g, \nabla, J_{ \pm}\right)$. 
Proof 1. We introduce the Weyl-Euler-Lagrange equations on a generalized (para)-Kählerian space form $\left(M, g, \nabla, J_{ \pm}\right)$using dynamic equation (15).

First of all, let $J_{ \pm}$almost generalized (para) product structure and by $\left(x_{i}, y_{i}\right)$ the coordinates of $M$. Also, on $\left(M, g, \nabla, J_{ \pm}\right)$, let semispray be a vector field given by

$$
\xi=X_{i} \frac{\partial}{\partial x_{i}}+Y_{i} \frac{\partial}{\partial y_{i}}, X_{i}=\dot{x}=y_{i}, Y_{i}=\dot{y}_{i}
$$

By Liouville vector field on a generalized (para)-Kählerian space form $\left(M, g, J_{ \pm}\right)$, we use the vector field determined by $V_{ \pm}=J_{ \pm}(\xi)$ and counted by

$$
V_{ \pm}=X_{i} e^{2 f} \frac{\partial}{\partial y_{i}} \pm Y_{i} e^{-2 f} \frac{\partial}{\partial x_{i}}
$$

Denote $T$ by the kinetic energy and $P$ by the potential energy of mechanics system on generalized (para)-Kählerian space of constant $J_{ \pm}$-sectional curvature. So, we write by $L=T-P$ a Lagrangian function and by $E_{L}=V(L)-L$ the energy function associated with $L$. Operator $i_{J_{ \pm}}$defined by $i_{J_{ \pm}}: \wedge^{2} M \rightarrow \wedge^{1} M$, is known as the interior product with $J_{ \pm}$, or sometimes the insertion operator, or contraction by $J_{ \pm}$. The exterior vertical derivation $d_{J_{ \pm}}$is determined by

$$
\mathbf{d}_{J_{ \pm}}=\left[i_{J_{ \pm}}, d\right]=i_{J_{ \pm}} d-d i_{J_{ \pm}}
$$

where $d$ is the usual exterior derivation. For an almost product structure $J_{ \pm}$determined by (11), the closed generalized (para)-Kählerian form is the 2 -form given by $\Phi_{L}=-d\left(\mathbf{d}_{J_{ \pm}} L\right)$ such that

$$
d_{J_{ \pm}}=e^{2 f} \frac{\partial}{\partial y_{i}} d x_{i} \pm e^{-2 f} \frac{\partial}{\partial x_{i}} d y_{i}, d_{J_{ \pm}}: \mathcal{F}(M) \rightarrow \wedge^{1} M
$$


Because of the closed generalized (para)-Kählerian form $\Phi_{L}$ on a generalized (para)-Kählerian space form $\left(M, g, \nabla, J_{ \pm}\right)$is a \pm symplectic structure,

$$
\begin{aligned}
\Phi_{L}= & 2 e^{2 f} \frac{\partial f}{\partial x_{i}} \frac{\partial L}{\partial y_{i}} d x_{i} \wedge d x_{i}+e^{2 f} \frac{\partial^{2} L}{\partial x_{i} \partial y_{i}} d x_{i} \wedge d x_{i} \\
\mp & 2 e^{-2 f} \frac{\partial f}{\partial x_{i}} \frac{\partial L}{\partial x_{i}} d y_{i} \wedge d x_{i} \pm e^{-2 f} \frac{\partial^{2} L}{\partial x_{i} \partial x_{i}} d y_{i} \wedge d x_{i} \\
& +2 e^{2 f} \frac{\partial f}{\partial y_{i}} \frac{\partial L}{\partial y_{i}} d x_{i} \wedge d y_{i}+e^{2 f} \frac{\partial^{2} L}{\partial y_{i} \partial y_{i}} d x_{i} \wedge d y_{j} \\
\mp & 2 e^{-2 f} \frac{\partial f}{\partial y_{i}} \frac{\partial L}{\partial x_{i}} d y_{i} \wedge d y_{j} \pm e^{-2 f} \frac{\partial^{2} L}{\partial y_{i} \partial x_{i}} d y_{i} \wedge d y_{i} .
\end{aligned}
$$

Therefore,

$$
\Phi_{L}(\xi)=\left[\begin{array}{c}
2 e^{2 f} \frac{\partial f}{\partial x_{i}} \frac{\partial L}{\partial y_{i}} X_{i} d x_{i}-2 e^{2 f} \frac{\partial f}{\partial x_{i}} \frac{\partial L}{\partial y_{i}} X_{i} d x_{i} \\
+e^{2 f} \frac{\partial^{2} L}{\partial x_{i} \partial y_{i}} X_{i} d x_{i}-e^{2 f} \frac{\partial^{2} L}{\partial x_{i} \partial y_{i}} X_{i} d x_{i} \\
\pm 2 e^{-2 f} \frac{\partial f}{\partial x_{i}} \frac{\partial L}{\partial x_{i}} X_{i} d y_{i} \mp e^{-2 f} \frac{\partial^{2} L}{\partial x_{i} \partial x_{i}} X_{i} d y_{i} \\
+2 e^{2 f} \frac{\partial f}{\partial y_{i}} \frac{\partial L}{\partial y_{i}} X_{i} d y_{i}+e^{2 f} \frac{\partial^{2} L}{\partial y_{i} \partial y_{i}} X_{i} d y_{i} \\
+2 e^{-2 f} \frac{\partial f}{\partial x_{i}} \frac{\partial L}{\partial x_{i}} Y_{i} d x_{i}+e^{-2 f} \frac{\partial^{2} L}{\partial x_{i} \partial x_{i}} Y_{i} d x_{i} \\
-2 e^{2 f} \frac{\partial f}{\partial y_{i}} \frac{\partial L}{\partial y_{i}} Y_{i} d x_{i}-e^{2 f} \frac{\partial^{2} L}{\partial y_{i} \partial y_{i}} Y_{i} d x_{i} \\
\mp 2 e^{-2 f} \frac{\partial f}{\partial y_{i}} \frac{\partial L}{\partial x_{i}} Y_{i} d y_{i} \pm 2 e^{-2 f} \frac{\partial f}{\partial y_{i}} \frac{\partial L}{\partial x_{i}} Y_{i} d y_{i} \\
\pm e^{-2 f} \frac{\partial^{2} L}{\partial y_{i} \partial x_{i}} Y_{i} d y_{i} \mp e^{-2 f} \frac{\partial^{2} L}{\partial y_{i} \partial x_{i}} Y_{i} d y_{i}
\end{array}\right]
$$


Energy of system is calculated as follows:

$$
E_{L}=V_{ \pm}(L)-L=X_{i} e^{2 f} \frac{\partial L}{\partial y_{i}} \pm Y_{i} e^{-2 f} \frac{\partial L}{\partial x_{i}}-L,
$$

and

$$
\begin{aligned}
d E_{L}= & X_{i} 2 e^{2 f} \frac{\partial f}{\partial x_{i}} \frac{\partial L}{\partial y_{i}} d x_{i}+X_{i} e^{2 f} \frac{\partial^{2} L}{\partial x_{i} \partial y_{i}} d x_{i} \\
\mp & 2 Y_{i} e^{-2 f} \frac{\partial f}{\partial x_{i}} \frac{\partial L}{\partial x_{i}} d x_{i} \pm Y_{i} e^{-2 f} \frac{\partial^{2} L}{\partial x_{i} \partial x_{i}} d x_{i}-\frac{\partial L}{\partial x_{i}} d x_{i} \\
& +X_{i} 2 e^{2 f} \frac{\partial f}{\partial y_{i}} \frac{\partial L}{\partial y_{i}} d y_{i}+X_{i} e^{2 f} \frac{\partial^{2} L}{\partial y_{i} \partial y_{i}} d y_{i} \\
\mp & Y_{i} 2 e^{-2 f} \frac{\partial f}{\partial y_{i}} \frac{\partial L}{\partial x_{i}} d y_{i} \pm Y_{i} e^{-2 f} \frac{\partial^{2} L}{\partial y_{i} \partial x_{i}} d y_{i}-\frac{\partial L}{\partial y_{i}} d y_{i} .
\end{aligned}
$$

By taking into account $i_{\xi} \Phi_{L}=d E_{L}$ and the curve $\alpha$, an integral curve of $\xi$, we obtain the equations determined by

$$
\begin{gathered}
-2 e^{2 f}\left[X_{i} \frac{\partial}{\partial x_{i}}+Y_{i} \frac{\partial}{\partial y_{i}}\right]\left(\frac{\partial L}{\partial y_{i}}\right)-e^{2 f}\left[X_{i} \frac{\partial}{\partial x_{i}}+Y_{i} \frac{\partial}{\partial y_{i}}\right]\left(\frac{\partial L}{\partial y_{i}}\right)+\frac{\partial L}{\partial x_{i}}=0, \\
\pm 2 e^{-2 f}\left[X_{i} \frac{\partial f}{\partial x_{i}}+Y_{i} \frac{\partial f}{\partial y_{i}}\right]\left(\frac{\partial L}{\partial x_{i}}\right) \mp e^{-2 f}\left[X_{i} \frac{\partial}{\partial x_{i}}+Y_{i} \frac{\partial}{\partial y_{i}}\right]\left(\frac{\partial L}{\partial x_{i}}\right)+\frac{\partial L}{\partial y_{i}}=0,
\end{gathered}
$$

or alternatively

$$
\begin{gathered}
-2 e^{2 f} \xi(f) \frac{\partial L}{\partial y_{i}}-e^{2 f} \xi\left(\frac{\partial L}{\partial y_{i}}\right)+\frac{\partial L}{\partial x_{i}}=0, \\
\pm 2 e^{-2 f} \xi(f) \frac{\partial L}{\partial x_{i}} \mp e^{-2 f} \xi\left(\frac{\partial L}{\partial x_{i}}\right)+\frac{\partial L}{\partial y_{i}}=0 .
\end{gathered}
$$


Also, by taking into account $\xi(\alpha(t))=\dot{\alpha}(t)=\frac{\partial \alpha}{\partial t}$ we get equations as follows:

$$
\begin{gathered}
-2 e^{2 f} \frac{\partial f}{\partial t} \frac{\partial L}{\partial y_{i}}-e^{2 f} \frac{\partial}{\partial t}\left(\frac{\partial L}{\partial y_{i}}\right)+\frac{\partial L}{\partial x_{i}}=0, \\
\pm 2 e^{-2 f} \frac{\partial f}{\partial t} \frac{\partial L}{\partial x_{i}} \mp e^{-2 f} \frac{\partial}{\partial t}\left(\frac{\partial L}{\partial x_{i}}\right)+\frac{\partial L}{\partial y_{i}}=0
\end{gathered}
$$

or shortly

$$
\begin{gathered}
\frac{\partial}{\partial t}\left(e^{2 f} \frac{\partial L}{\partial y_{i}}\right)-\frac{\partial L}{\partial x_{i}}=\mathbf{0} \\
\frac{\partial}{\partial t}\left(e^{-2 f} \frac{\partial L}{\partial x_{i}}\right) \mp \frac{\partial L}{\partial y_{i}}=\mathbf{0}
\end{gathered}
$$

such that the differential equations (19) are named conformal Weyl-EulerLagrange equations on a generalized (para)-Kählerian space form $\left(M, g, \nabla, J_{ \pm}\right)$. Finally, it may be said that the triple $\left(M, \Phi_{L}, \xi\right)$ is conformal Weyl-Euler-Lagrange mechanical system on generalized (para)Kählerian manifold of constant $J_{ \pm}$-sectional curvature $\left(M, g, \nabla, J_{ \pm}\right)$.

Proof 2. Let's use the proposed Theorem 4 for the second proof.

(1) First, let's consider holomorphic structures (11).

$$
J_{ \pm}\left(\frac{\partial}{\partial x_{i}}\right)=e^{2 f} \frac{\partial}{\partial y_{i}}, \quad J_{ \pm}\left(\frac{\partial}{\partial y_{i}}\right)= \pm e^{-2 f} \frac{\partial}{\partial x_{i}} .
$$

(2) " $L$ " of the Lagrangian function is added to derivative expressions,

$$
J_{ \pm}\left(\frac{\partial L}{\partial x_{i}}\right)=e^{2 f} \frac{\partial L}{\partial y_{i}}, \quad J_{ \pm}\left(\frac{\partial L}{\partial y_{i}}\right)= \pm e^{-2 f} \frac{\partial L}{\partial x_{i}}
$$

(3) The places of the derivative expressions on both sides of the equality are completely displaced.

$$
J_{ \pm}\left(e^{2 f} \frac{\partial L}{\partial y_{i}}\right)=\frac{\partial L}{\partial x_{i}}, \quad J_{ \pm}\left( \pm e^{-2 f} \frac{\partial L}{\partial x_{i}}\right)=\frac{\partial L}{\partial y_{i}} .
$$


(4) In this step, $\left(\frac{\partial}{\partial t}\right)$ is written instead of $J_{ \pm}$,

$$
\frac{\partial}{\partial t}\left(e^{2 f} \frac{\partial L}{\partial y_{i}}\right)=\frac{\partial L}{\partial x_{i}}, \quad \frac{\partial}{\partial t}\left( \pm e^{-2 f} \frac{\partial L}{\partial x_{i}}\right)=\frac{\partial L}{\partial y_{i}} .
$$

(5) If the distribution property is applied here, the equations are found as follows:

$$
\frac{\partial}{\partial t}\left(e^{2 f} \frac{\partial L}{\partial y_{i}}\right)-\frac{\partial L}{\partial x_{i}}=0, \quad \frac{\partial}{\partial t}\left(e^{-2 f} \frac{\partial L}{\partial x_{i}}\right) \mp \frac{\partial L}{\partial y_{i}}=0 .
$$

So the proof is complete.

\section{Weyl-Hamiltonian Mechanics Systems}

Theorem 5. Let $J$ be a para-complex or complex structures on the tangent space $T_{p}(M)$. Let $J^{*}$ be which is dual to J. $J^{*}$ be defined that a para-complex or complex structures of dual $J$, a Liouville form and a 1-form on definition manifolds are indicated by $J^{*}, \Omega$ and $\omega$, respectively. If the dual endomorphism $J^{*}$ of the cotangent space and Liouville form $\Omega=J^{*}(\omega)$, then Hamilton motion equations can be found as follows: Hamiltonian motion equations for $\lambda=\lambda(x, y)=\Omega(x, y) d x+\Psi(x, y) d y$ are

$$
\begin{aligned}
& \frac{d x}{d t}=\frac{1}{\partial_{y} \Omega-\partial_{x} \Psi} \frac{\partial H}{\partial y}, \\
& \frac{d y}{d t}=\frac{-1}{\partial_{y} \Omega-\partial_{x} \Psi} \frac{\partial H}{\partial x} .
\end{aligned}
$$

For example: Base structures are (1) $J^{*}\left(z_{i}\right)=-\mathbf{j} d z_{i}$ and (2) $J^{*}\left(\bar{z}_{i}\right)=\mathbf{j} d \bar{z}_{i}$ for $\lambda=J^{*}(\omega)=\frac{1}{2} \mathbf{j}\left(z_{i} d \bar{z}_{i}-\bar{z}_{i} d z_{i}\right), \Omega=\frac{1}{2} \mathbf{j} z_{i}, \Psi=-\frac{1}{2} \mathbf{j} \bar{z}_{i}, \partial_{z_{i}} \Omega=\frac{1}{2} \mathbf{j}, \partial_{\bar{z}_{i}}$ $\Omega=-\frac{1}{2} \mathbf{j}, \partial_{y} \Omega-\partial_{x} \Psi=\mathbf{j}, \mathbf{j}^{2}=1$. Equations are (1) $\frac{d z_{i}}{d t}=-\mathbf{j} \frac{d H}{d \bar{z}_{i}}$ and (2) $\frac{d \bar{z}_{i}}{d t}=\mathbf{j} \frac{d H}{d z_{i}}[4]$. 
Example. In this study, Weyl-Hamilton equations are on a generalized (para)-Kählerian space form $\left(M, g, \nabla, J_{ \pm}^{*}\right)$ using Theorem 5 for

$$
\begin{aligned}
\Omega_{ \pm} & =-\frac{1}{2}\left(-e^{2 f} x_{i} d y_{i} \pm e^{-2 f} y_{i} d x_{i}\right) \\
& =\frac{1}{2}\left(\mp e^{-2 f} y_{i} d x_{i}+e^{2 f} x_{i} d y_{i}\right)
\end{aligned}
$$

$$
\begin{aligned}
& \frac{d x_{i}}{d t}=\frac{-2}{\left[e^{2 f}+2 x_{i} \frac{\partial f}{\partial x_{i}}+e^{-2 f}-2 x_{i} \frac{\partial f}{\partial y_{i}}\right]} \frac{\partial H}{\partial y_{i}}, \\
& \frac{d y_{i}}{d t}=\frac{2}{\left[e^{2 f}+2 x_{i} \frac{\partial f}{\partial x_{i}}+e^{-2 f}-2 x_{i} \frac{\partial f}{\partial y_{i}}\right]} \frac{\partial H}{\partial x_{i}} .
\end{aligned}
$$

Hence, the differential equations presented in (39) are named conformal Weyl-Hamilton equations on the almost pseudo/para-Kähler Weyl manifolds $\left(M, g, \nabla, J_{ \pm}^{*}\right)$. After that the triple $\left(M, \Phi_{ \pm}, X_{H}\right)$ is said to be a conformal Weyl-Hamiltonian mechanical system on $\left(M, g, \nabla, J_{ \pm}^{*}\right)$.

Proof 1. We provide using the dynamical equation (17) with information about Weyl-Hamilton equations on a generalized (para)Kählerian space form $\left(M, g, \nabla, J_{ \pm}^{*}\right)$, of constant $J_{ \pm}^{*}$-sectional curvature:

$$
\begin{aligned}
& J_{ \pm}^{*}\left(d x_{i}\right)=e^{2 f} d y_{i}, \\
& J_{ \pm}^{*}\left(d y_{i}\right)= \pm e^{-2 f} d x_{i} .
\end{aligned}
$$


Let $J_{ \pm}^{*}$ be an almost generalized (para)-product structure defined by (12) and $\Omega_{ \pm}$Liouville form determined by

$$
\Omega_{ \pm}=J_{ \pm}^{*}\left(\omega_{ \pm}\right)=-\frac{1}{2}\left(-e^{2 f} x_{i} d y_{i} \pm e^{-2 f} y_{i} d x_{i}\right)
$$

such that

$$
\omega_{ \pm}=-\frac{1}{2}\left(-x_{i} d x_{i} \pm y_{i} d y_{i}\right)
$$

is a 1 -form on $M$ [29], where $y_{i}=\frac{\partial L}{\partial \dot{x}_{i}}$. If $\Phi_{ \pm}=-d \Omega_{ \pm}$is a generalized closed (para)-Kählerian form, then it is also a generalized (para)symplectic structure on $M$.

Let $\left(M, g, \nabla, J_{ \pm}^{*}\right)$ be a generalized (para)-Kählerian space form, of constant $J_{ \pm}^{*}$-sectional curvature, with a generalized closed (para)Kählerian form $\Phi_{ \pm}$.

Let us think that a Hamiltonian vector field $X_{H}$ associated with Hamiltonian energy $H$ is given by

$$
X_{H}=X_{i} \frac{\partial}{\partial x_{i}}+Y_{i} \frac{\partial}{\partial y_{i}} .
$$

For the generalized closed (para)-Kählerian form $\Phi_{ \pm}$on $M$, we have

$$
\begin{aligned}
\Phi_{ \pm}=-\frac{1}{2} d\left(x_{i} e^{2 f} d y_{i} \pm y_{i} e^{-2 f} d x_{i}\right) \\
=-\frac{1}{2}\left\{\begin{array}{c}
e^{2 f} \frac{\partial x_{i}}{\partial x_{j}} d x_{j} \wedge d y_{i}+2 x_{i} e^{2 f} \frac{\partial f}{\partial x_{j}} d x_{j} \wedge d y_{i} \\
\pm e^{-2 f} \frac{\partial y_{i}}{\partial x_{j}} d x_{j} \wedge d x_{i} \mp 2 y_{i} e^{-2 f} \frac{\partial f}{\partial x_{j}} d x_{j} \wedge d x_{i} \\
+e^{2 f} \frac{\partial x_{i}}{\partial y_{j}} d y_{j} \wedge d y_{i}+2 x_{i} e^{2 f} \frac{\partial f}{\partial y_{j}} d y_{j} \wedge d y_{i} \\
\pm e^{-2 f} \frac{\partial y_{i}}{\partial y_{j}} d y_{j} \wedge d x_{i} \mp 2 y_{i} e^{-2 f} \frac{\partial f}{\partial y_{j}} d y_{j} \wedge d x_{i}
\end{array}\right\} .
\end{aligned}
$$


Then it goes like the following:

$$
\begin{aligned}
& i_{X_{H}} \Phi_{ \pm}=\Phi_{ \pm}\left(X_{H}\right) \\
& =-\frac{1}{2}\left\{\begin{array}{c}
e^{2 f} X_{i}\left[d x_{i} \frac{\partial}{\partial x_{i}} d y_{i}-d y_{i} \frac{\partial}{\partial x_{i}} d x_{i}\right] \\
+2 x_{i} e^{2 f} \frac{\partial f}{\partial x_{i}} X_{i}\left[d x_{i} \frac{\partial}{\partial x_{i}} d y_{i}-d y_{i} \frac{\partial}{\partial x_{i}} d x_{i}\right] \\
\pm e^{-2 f} X_{i}\left[d y_{i} \frac{\partial}{\partial x_{i}} d x_{i}-d x_{i} \frac{\partial}{\partial x_{i}} d y_{i}\right] \\
\mp 2 x_{i} e^{-2 f} \frac{\partial f}{\partial y_{i}} X_{i}\left[d y_{i} \frac{\partial}{\partial x_{i}} d x_{i}-d x_{i} \frac{\partial}{\partial x_{i}} d y_{i}\right] \\
e^{2 f} Y_{i}\left[d x_{i} \frac{\partial}{\partial y_{i}} d y_{i}-d y_{i} \frac{\partial}{\partial y_{i}} d x_{i}\right] \\
+2 x_{i} e^{2 f} \frac{\partial f}{\partial x_{i}} Y_{i}\left[d x_{i} \frac{\partial}{\partial y_{i}} d y_{i}-d y_{i} \frac{\partial}{\partial y_{i}} d x_{i}\right] \\
\pm e^{-2 f} Y_{i}\left[d y_{i} \frac{\partial}{\partial y_{i}} d x_{i}-d x_{i} \frac{\partial}{\partial y_{i}} d y_{i}\right] \\
\mp 2 x_{i} e^{-2 f} \frac{\partial f}{\partial y_{i}} Y_{i}\left[d y_{i} \frac{\partial}{\partial y_{i}} d x_{i}-d x_{i} \frac{\partial}{\partial y_{i}} d y_{i}\right]
\end{array}\right\} .
\end{aligned}
$$

From (45) with respect to $i_{X_{H}} \Phi_{ \pm}=d H$, we obtain generalized (para)Hamiltonian vector field on generalized (para)-Kählerian space of constant $J_{ \pm}^{*}$-sectional curvature.

Let $\left(M, g, \nabla, J_{ \pm}^{*}\right)$ be a generalized (para)-Kählerian space form, of constant $J_{ \pm}^{*}$-sectional curvature, with a generalized closed (para)Kählerian form $\Phi_{ \pm}$. Moreover, the differential of Hamiltonian energy $H$ is gained by

$$
d H=\frac{\partial H}{\partial x_{i}} d x_{i}+\frac{\partial H}{\partial y_{i}} d y_{i}
$$


Using the equation $i_{X_{H}} \Phi=d H$, the following statement is reached:

$$
-\frac{1}{2}\left\{\begin{array}{c}
e^{2 f} X_{i} d y_{i}+2 x_{i} e^{2 f} \frac{\partial f}{\partial x_{i}} X_{i} d y_{i} \\
\pm e^{-2 f} X_{i} d y_{i} \mp 2 x_{i} e^{-2 f} \frac{\partial f}{\partial y_{i}} X_{i} d y_{i} \\
-e^{2 f} Y_{i} d x_{i}-2 x_{i} e^{2 f} \frac{\partial f}{\partial x_{i}} Y_{i} d x_{i} \\
\pm e^{-2 f} Y_{i} d x_{i} \mp 2 x_{i} e^{-2 f} \frac{\partial f}{\partial y_{i}} Y_{i} d x_{i}
\end{array}\right\}=\frac{\partial H}{\partial x_{i}} d x_{i}+\frac{\partial H}{\partial y_{i}} d y_{i}
$$

So, a generalized (para)-Hamiltonian vector field $X_{H}$ associated with Hamiltonian energy $H$ is decided by using (17), the Hamilton vector field is calculated

$$
\begin{aligned}
& X_{i}=\frac{-2}{\left[e^{2 f}+2 x_{i} e^{2 f} \frac{\partial f}{\partial x_{i}} \pm e^{-2 f} \mp 2 x_{i} e^{-2 f} \frac{\partial f}{\partial y_{i}}\right]} \frac{\partial H}{\partial y_{i}}, \\
& Y_{i}=\frac{2}{\left[e^{2 f}+2 x_{i} e^{2 f} \frac{\partial f}{\partial x_{i}} \pm e^{-2 f} \mp 2 x_{i} e^{-2 f} \frac{\partial f}{\partial y_{i}}\right]} \frac{\partial H}{\partial x_{i}} .
\end{aligned}
$$

Let $\alpha: I \subset \mathbb{R} \rightarrow M$ be an integral curve of a generalized (para)Hamiltonian vector field $X_{H}$, i.e., $X_{H}(\alpha(t))=\dot{\alpha}, t \in I$.

$$
\begin{aligned}
X_{H}= & \frac{-2}{\left[e^{2 f}+2 x_{i} e^{2 f} \frac{\partial f}{\partial x_{i}} \pm e^{-2 f} \mp 2 x_{i} e^{-2 f} \frac{\partial f}{\partial y_{i}}\right]} \frac{\partial H}{\partial y_{i}} \frac{\partial}{\partial x_{i}} \\
& +\frac{2}{\left[e^{2 f}+2 x_{i} e^{2 f} \frac{\partial f}{\partial x_{i}} \pm e^{-2 f} \mp 2 x_{i} e^{-2 f} \frac{\partial f}{\partial y_{i}}\right]} \frac{\partial H}{\partial x_{i}} \frac{\partial}{\partial y_{i}} .
\end{aligned}
$$


In the local coordinates we have $\alpha(t)=\left(x_{i}(t), y_{i}(t)\right)$, and

$$
\begin{gathered}
\dot{\alpha}(t)=\frac{\partial \alpha}{\partial t}=\frac{d x_{i}}{d t} \frac{\partial}{\partial x_{i}}+\frac{d y_{i}}{d t} \frac{\partial}{\partial y_{i}} . \\
\frac{-2}{\left[e^{2 f}+2 x_{i} e^{2 f} \frac{\partial f}{\partial x_{i}} \pm e^{-2 f} \mp 2 x_{i} e^{-2 f} \frac{\partial f}{\partial y_{i}}\right]} \frac{\partial H}{\partial y_{i}} \frac{\partial}{\partial x_{i}} \\
+\frac{2}{\left[e^{2 f}+2 x_{i} e^{2 f} \frac{\partial f}{\partial x_{i}} \pm e^{-2 f} \mp 2 x_{i} e^{-2 f} \frac{\partial f}{\partial y_{i}}\right]} \frac{\partial H}{\partial x_{i}} \frac{\partial}{\partial y_{i}} \\
=\frac{d x_{i}}{d t} \frac{\partial}{\partial x_{i}}+\frac{d y_{i}}{d t} \frac{\partial}{\partial y_{i}} .
\end{gathered}
$$

We infer the equations so-called conformal equations:

$$
\begin{aligned}
& \frac{d x_{i}}{d t}=\frac{-2}{\left[e^{2 f}+2 x_{i} e^{2 f} \frac{\partial f}{\partial x_{i}} \pm e^{-2 f} \mp 2 x_{i} e^{-2 f} \frac{\partial f}{\partial y_{i}}\right]} \frac{\partial H}{\partial y_{i}}, \\
& \frac{d y_{i}}{d t}=\frac{2}{\left[e^{2 f}+2 x_{i} e^{2 f} \frac{\partial f}{\partial x_{i}} \pm e^{-2 f} \mp 2 x_{i} e^{-2 f} \frac{\partial f}{\partial y_{i}}\right]} \frac{\partial H}{\partial x_{i}} .
\end{aligned}
$$

Proof 2. We use Theorem 5 for proof $[\lambda=\lambda(x, y)=\Omega(x, y) d x+\Psi$ $(x, y) d y$, equations are (1) $\frac{d x}{d t}=\frac{1}{\partial_{y} \Omega-\partial_{x} \Psi} \frac{\partial H}{\partial y}$ and (2) $\frac{d y}{d t}=\frac{-1}{\partial_{y} \Omega-\partial_{x} \Psi}$ $\left.\frac{\partial H}{\partial x}\right]$. The following steps are followed for proof:

(1) $\lambda_{ \pm}=-\frac{1}{2}\left(-e^{2 f} x_{i} d y_{i} \pm e^{-2 f} y_{i} d x_{i}\right)$

$$
=\frac{1}{2}\left(\mp e^{-2 f} y_{i} d x_{i}+e^{2 f} x_{i} d y_{i}\right),
$$

(2) $\Omega=\mp \frac{1}{2} e^{-2 f} y_{i}$ and $\Psi=\frac{1}{2} e^{2 f} x_{i}$, 
(3) $\partial_{y} \Omega=\frac{1}{2}\left(\mp e^{-2 f} \pm 2 y_{i} e^{-2 f} \frac{\partial f}{\partial y_{i}}\right)$,

$$
\partial_{x} \Psi=\frac{1}{2}\left(e^{2 f}+2 e^{2 f} x_{i} \frac{\partial f}{\partial x_{i}}\right),
$$

(4) $\partial_{y} \Omega-\partial_{x} \Psi=\frac{1}{2}\left(\mp e^{-2 f} \pm 2 e^{-2 f} y_{i} \frac{\partial f}{\partial y_{i}}\right)$

$$
\begin{gathered}
-\frac{1}{2}\left(e^{2 f}+2 e^{2 f} x_{i} \frac{\partial f}{\partial x_{i}}\right) \\
=\frac{1}{2}\left(\mp e^{-2 f} \pm 2 e^{-2 f} y_{i} \frac{\partial f}{\partial y_{i}}-e^{2 f}-2 e^{2 f} x_{i} \frac{\partial f}{\partial x_{i}}\right),
\end{gathered}
$$

(5) $\frac{d x}{d t}=\frac{1}{\partial_{y} \Omega-\partial_{x} \Psi} \frac{\partial H}{\partial y}$

$$
=\frac{1}{\frac{1}{2}\left[\mp e^{-2 f} \pm 2 e^{-2 f} y_{i} \frac{\partial f}{\partial y_{i}}-e^{2 f}-2 e^{2 f} x_{i} \frac{\partial f}{\partial x_{i}}\right]} \frac{\partial H}{\partial y},
$$

(6) $\frac{d y}{d t}=\frac{-1}{\partial_{y} \Omega-\partial_{x} \Psi} \frac{\partial H}{\partial x}$

$$
=\frac{-1}{\frac{1}{2}\left[\mp e^{-2 f} \pm 2 e^{-2 f} y_{i} \frac{\partial f}{\partial y_{i}}-e^{2 f}-2 e^{2 f} x_{i} \frac{\partial f}{\partial x_{i}}\right]} \frac{\partial H}{\partial y},
$$

Thus, the following equations are reached:

$$
\begin{aligned}
& \frac{d x_{i}}{d t}=\frac{-2}{\left[e^{2 f}+2 x_{i} e^{2 f} \frac{\partial f}{\partial x_{i}} \pm e^{-2 f} \mp 2 x_{i} e^{-2 f} \frac{\partial f}{\partial y_{i}}\right]} \frac{\partial H}{\partial y_{i}}, \\
& \frac{d y_{i}}{d t}=\frac{2}{\left[e^{2 f}+2 x_{i} e^{2 f} \frac{\partial f}{\partial x_{i}} \pm e^{-2 f} \mp 2 x_{i} e^{-2 f} \frac{\partial f}{\partial y_{i}}\right]} \frac{\partial H}{\partial x_{i}} .
\end{aligned}
$$

So the proof is complete. 


\section{Conclusion}

We derived the following conclusions:

(1) By using Weyl metric (7) the base structures given in (9) and (10) should be transferred to (11) and (12).

(2) Conformal Weyl-Euler-Lagrange equations or partial differential equations (19) found by using the Theorem 4 with a generalized (para)Kählerian space form $\left(M, g, \nabla, J_{ \pm}\right)$.

(3) Conformal Weyl-Hamilton equations or partial differential equations (39) found by using the Theorem 5 with a generalized (para)Kählerian space form $\left(M, g, \nabla, J_{ \pm}^{*}\right)$.

(4) It is seen that equations can be found without errors and without need to use the dynamic equations (15) and (17) for calculation of movement equations by these theorems.

(5) In this way, researchers can find the Lagrangian and Hamiltonian motion equations on any space/manifold with Theorems 4 and 5 correctly without need calculating using long operations.

(6) In future studies, researchers may be advised to use Theorems 4 and 5 for to know the route and their requirements of mechanical systems in defined space/manifold.

\section{Discussion}

(1) The geodesics of objects moving on the almost pseudo/para-Kähler Weyl manifolds were modelled using differential equations (19) and (39). These geodesics can be used for the positions and requirements of objects moving in space by the time.

(2) This study raised geodesics for about the non-linear orbits of the objects in the space by the help of revised features using Weyl's unified theorem on the almost pseudo/para-Kähler Weyl manifolds. 
(3) The obtained equations are very important to explain problems about physics and other related fields. Because in Weyl's unified theory, the metrics are interpreted as the gravitational potential, as in general relativity, and the corresponding forms are interpreted as the electromagnetic potentials [31].

(4) In future studies, researchers may be advised an explanation of the rotational spatial mechanical-physical problems on the almost pseudo/para-Kähler Weyl manifolds.

\section{Acknowledgements}

This work was supported by the agency BAP of Pamukkale University, Project number: 1217. In addition, this study was presented orally at the International Congress in Honor of Professor Hari M. Savastava at The Auditorium at the Campus of Uludag University, August 23-26, 2012, Bursa-TURKEY.

\section{References}

[1] P. Gilkey and S. Nikčević, (Para)-Kähler Weyl structures, (2012), 1-14, arXiv:1204.0724.

[2] M. de León and P. R. Rodrigues, Generalized Classical Mechanics and Field Theory, North-Holland Mathematics Studies, 112, North-Holland, Amsterdam, 2000.

[3] H. de Vries, Understanding Relativistic Quantum Field Theory, The Hamiltonian and Lagrangian Densities, Chapter 22 (2009).

[4] M. Tekkoyun, On para-Euler-Lagrange and para-Hamiltonian equations, Physics Letters A 340(1-4) (2005), 7-11.

DOI: https://doi.org/10.1016/j.physleta.2005.03.055

[5] M. Tekkoyun and M. Sari, Bi-para-mechanical systems on the bi-Lagrangian manifold, Physica B: Condensed Matter 405(10) (2010), 2390-2393.

DOI: https://doi.org/10.1016/j.physb.2010.02.052

[6] M. Tekkoyun and Y. Yayli, Mechanical systems on generalized-quaternionic Kähler manifolds, International Journal of Geometric Methods in Modern Physics 8(7) (2011), 1419.1431.

DOI: https://doi.org/10.1142/S0219887811005804 
[7] P. Gilkey and S. Nikčević, 4-Dimensional (para)-Kähler-Weyl structures, (2012), 1-8, arXiv:1210.6769.

[8] G. Ganchev and V. Mihova, Warped product Kähler manifolds and Bochner-Kähler metrics, Journal of Geometry and Physics 58(7) (2008), 803-824.

DOI: https://doi.org/10.1016/j.geomphys.2008.02.002

[9] C. L. Bejan and N. C. Chiriac, Weyl structures on almost paracontact manifolds, International Journal of Geometric Methods in Modern Physics 10(1) (2013); Article 1220019 .

DOI: https://doi.org/10.1142/S0219887812200198

[10] D. Cherney, E. Latini and A. Waldron, $(p, q)$-form Kähler electromagnetism, (2009), 1-5, arXiv:0901.3788.

[11] X. Sun and Y. Wang, Geometric Schrödinger-airy flows on Kähler manifolds, (2012), 1-35, arXiv:1203.0549.

[12] D. S. Freed, Special Kähler manifolds, Communications in Mathematical Physics 203(1) (1999), 31-52.

DOI: https://doi.org/10.1007/s002200050604

[13] N. Tyurin, Hamiltonian dynamics on the moduli space of half weighted BohrSommerfeld Lagrangian subcycles of a fixed volume, (2000), 1-11, arXiv:math/0010104.

[14] S. L. Lyakhovich and A. A. Sharapov, Kähler polarization and Wick quantization of Hamiltonian systems subject to second-class constraints, Modern Physics Letters A 17(2) (2002), 121-129.

DOI: https://doi.org/10.1142/S0217732302006230

[15] Z. Rongye, Newtonian mechanics on Kähler manifold, Applied Mathematics and Mechanics 17(8) (1996), 751-764.

DOI: https://doi.org/10.1007/BF00189563

[16] Z. Kasap and M. Tekkoyun, Mechanical systems on almost para/pseudo-KählerWeyl manifolds, International Journal of Geometric Methods in Modern Physics 10(5) (2013); Article 1350008.

DOI: https://doi.org/10.1142/S0219887813500084

[17] M. Z. Petrović and L. S. Velimirović, A new type of generalized para-Kähler spaces and holomorphically projective transformations, Bulletin of the Iranian Mathematical Society 45(4) (2019), 1021-1043.

DOI: https://doi.org/10.1007/s41980-018-0182-y

[18] M. Z. Petrović, Holomorphically projective mappings between generalized hyperbolic Kähler spaces, Journal of Mathematical Analysis and Applications 447(1) (2017), 435-451.

DOI: https://doi.org/10.1016/j.jmaa.2016.10.016 
[19] Josef Mikeš, Elena Stepanova and Alena Vanžurová, Differential Geometry of Special Mappings, Palacký University, Olomouc, 2015.

[20] G. B. Folland, Weyl manifolds, Journal of Differential Geometry 4(2) (1970), 145-153.

DOI: https://doi.org/10.4310/jdg/1214429379

[21] P. Gilkey and S. Nikčević, Kähler-Weyl manifolds of dimension, 4 (2011), 1-10, arXiv:1109.4532.

[22] L. Kadosh, Topics in Weyl Geometry, Dissertational, University of California, 1996.

[23] H. Weyl, Space-Time-Matter, Dover Publications 1922, Translated from the $4^{\text {th }}$ German edition by H. Brose, London: Methuen, Reprint New York, Dover, 1952.

[24] M. Z. Petrović, Generalized para-Kähler spaces in Eisenhart's sense admitting a holomorphically projective mapping, Filomat 33(13) (2019), 4001-4012.

\section{DOI: https://doi.org/10.2298/FIL1913001P}

[25] M. Hazewinkel, Encyclopaedia of Mathematics: Fibonacci Method-H, Kluwer Academic Publishers, 1989.

DOI: https://doi.org/10.1007/978-94-009-5997-2

[26] P. Gilkey and S. Nikčević, Kähler and para-Kähler curvature Weyl manifolds, (2010), 1-11, arXiv:1011.4844.

[27] J. Klein, Escapes variationnels et mécanique, Annales de l'Institut Fourier 12 (1962), 1-24.

$$
\text { DOI: https://doi.org/10.5802/aif.120 }
$$

[28] M. Brozos-Vazquez, P. Gilkey and S. Nikčević, Geometric realizations of affine Kähler curvature models, Results in Mathematics 59(3) (2011), 507-521.

DOI: https://doi.org/10.1007/s00025-011-0105-1

[29] R. Miron, D. Hrimiuc, H. Shimada and S. V. Sabau, The Geometry of Hamilton and Lagrange Spaces, Kluwer Academic Publishers, FTPH, 118 (2001).

[30] M. de Leon and P. R. Rodrigues, Methods of Differential Geometry in Analytical Mechanics, North-Holland Math. St., 152, Elsevier Sc. Pub. Com. Inc., Amsterdam, 1989.

[31] B. Hornberger, Electric and Magnetic Forces in Lagrangian and Hamiltonian Formalism, Lecture Notes, 2001. 